\title{
Coupled-channel analysis of deuteron scattering on ${ }^{56} \mathrm{Fe}$
}

\author{
Yujie Liu ${ }^{1}$, Tao $\mathrm{Ye}^{2, *}$, Weili $\mathrm{Sun}^{2}$, and Hairui $\mathrm{Guo}^{2}$ \\ ${ }^{1}$ Graduate School of China Academy of Engineering Physics \\ ${ }^{2}$ Institute of Applied Physics and Computational Mathematics
}

\begin{abstract}
The deuteron elastic and inelastic scattering on ${ }^{56} \mathrm{Fe}$ is calculated by using the coupled-channel method and the collective motion models. Based on the experimental angular distributions of scattering processes, an optimal set of parameters are obtained for the deuteron's coupled-channel optical model potential and ${ }^{56} \mathrm{Fe}$ 's deformation including first ten low-lying excited states. The calculation results are in good agreement with the experimental data at low incident energies $\left(E_{d}<30 \mathrm{MeV}\right)$, except for $4_{1}^{+}(2.085 \mathrm{MeV})$ and $2_{3}^{+}(2.960$ $\mathrm{MeV})$, and the deviation is found for several states at $56 \mathrm{MeV}$. That indicates further analyses and discussions are necessary.
\end{abstract}

\section{Introduction}

Deuteron-induced reaction catches much attention recently in association with applications for two main purposes. One is the fusion energy technology, and the other is the intense neutron source. Many scientific facilities are served for these applications around the world now, such as ITER [1], IFMIF [2] and CiADS [3], and so on. In these facilities, nuclear data including deuteron scattering angular distribution and neutron production cross section is essential for the design and operation activities. For example, deuteron-induced reaction data on the structure material like iron were used for the safety and radioprotection calculations of facilities for the IFMIF/EVEDA activities [4]. Therefore, the reliable nuclear data are requested for the safety of the facilities.

The input parameters of the theoretical models, like the optical model potential (OMP) and the collective motion model, are important to analyze the nuclear scattering processes and reactions. The accurate parameters can lead to the reliable nuclear data. As the first step in the nuclear data evaluation, it is especially crucial to get the proper OMP parameters because OMP provides the distorted waves implanted in the calculations of many nuclear reactions.

There are two kinds of OMPs, spherical (or singlechannel) OMP and coupled-channel (CC) OMP. Usually, the spherical OMP parameters are obtained by fitting the experimental data of the elastic scattering. Two new deuteron global OMPs [5, 6] are developed both in 2006. For incident energies up to $183 \mathrm{MeV}$ and target nuclei ranging from ${ }^{12} \mathrm{C}$ to ${ }^{238} \mathrm{U}$, An and Cai [5] obtained a set of global deuteron optical potential parameters which fitted well the experimental data. The similar work also has been done by Han et al. [6]. However, the CC-OMP is more theoretically consistent than spherical OMP for

*e-mail: ye_tao@iapcm.ac.cn both elastic and inelastic scattering processes, and it is useful to reduce the ambiguity and improve the accuracy of parameters. By considering both the deuteron OMP for deuteron-target interaction and the collective motion model for the deformation of target nucleus, the CC-OMP describes more consistently the angular distributions of target's low-lying excited states and utilizes more information from inelastic scattering, especially for the excited states coupled strongly to the ground state (G.S.)

In this work, the coupled channel method was employed to calculate deuteron scattering angular distribution for ${ }^{56} \mathrm{Fe}$ 's ground state and several low-lying excited states. The asymmetric rotational model and vibrational model were employed to describe these collective lowlying states. The calculations were carried out by using ECIS code [7].

\section{Theoretical models}

The coupled-channel method needs the optical model describing the deuteron-target interaction and the collective motion models describing the target deformation. At first, the deuteron OMP used by An and Cai [5] are chosen, which includes a Woods-Saxon form for the real and the imaginary volume terms, and a derivative WoodsSaxon form for the imaginary surface term, and a Thomas form for the real and imaginary spin-orbital terms. The Coulomb term $V_{c}$ is also included and taken as a potential of uniformly charged sphere with radius $R_{c}$. The phenomenological optical model potential form is given as follows:

$$
\begin{aligned}
V(r)= & -V_{R} f_{R}(r)-i W_{V} f_{V}(r)+i 4 a_{S} W_{S} \frac{d f_{S}(r)}{d r} \\
& +\lambda_{\pi}^{2} \frac{V_{S O}+i W_{S O}}{r} \frac{f_{S O}(r)}{d r} \vec{\sigma} \cdot \vec{l}+V_{C}(r) .
\end{aligned}
$$




$$
V_{C}(r)= \begin{cases}\frac{Z z e^{2}}{2 R_{C}}\left(3-\frac{r^{2}}{R_{C}^{2}}\right) & r \leqslant R_{C}, \\ \frac{Z z e^{2}}{r} & r \geqslant R_{C} .\end{cases}
$$

The Woods-Saxon form factor is given as

$$
\begin{aligned}
f\left(r, r_{x}, a_{x}\right) & =\frac{1}{1+\exp \left(\frac{r-R_{i}}{a_{i}}\right)}, \quad \text { with } i=\mathrm{R}, \mathrm{V}, \mathrm{S}, \mathrm{SO}, \\
R_{i} & =r_{i} A^{1 / 3}, \quad \text { with } i=\mathrm{R}, \mathrm{V}, \mathrm{S}, \mathrm{SO}, \mathrm{C},
\end{aligned}
$$

where the symbols R, V, S, SO and C denote the real and imaginary volume terms, imaginary surface term, spinorbit and Coulomb terms, respectively.

Secondly, the asymmetric rotational model and the vibration model embodied in ECIS code [7] are employed to describe the ground state rotational band and other lowlying collective states, respectively. For some vibrational nuclei including ${ }^{56} \mathrm{Fe}$, a dispersive coupled-channel OMP with soft-rotator couplings by $\mathrm{Li}$ et al. [8] showed rather good descriptions of the neutron and proton scattering. However, there is no nuclear reaction code available using the same model as Li for deuteron scattering. The ECIS code is thus selected and both above models are used.

We use asymmetric rotational model to describe the first three levels of the ground state rotational band, i.e., $J^{\pi}=0_{1}^{+}$(G.S.), $2^{+}(0.845 \mathrm{MeV}), 4_{1}^{+}(2.085 \mathrm{MeV})$. In the asymmetric rotational model, the radius is given by:

$$
\begin{aligned}
R(\theta) & =R_{0}\left\{1+\beta_{2}\left[\cos \gamma Y_{2}^{0}(\theta)+\frac{1}{\sqrt{2}} \sin \gamma\left(Y_{2}^{2}(\theta)+Y_{2}^{-2}(\theta)\right)\right]\right. \\
& +\beta_{4} \cos \gamma^{\prime} Y_{4}^{0}(\theta)+\beta_{4} \sin \gamma^{\prime}\left[\frac{1}{\sqrt{2}} \cos \delta\left(Y_{4}^{2}(\theta)+Y_{4}^{-2}(\theta)\right)\right. \\
& \left.\left.+\frac{1}{\sqrt{2}} \sin \delta\left(Y_{4}^{4}(\theta)+Y_{4}^{-4}(\theta)\right)\right]+\ldots\right\}
\end{aligned}
$$

The deformation parameters in the above formula are given in Table 1, which are derived from Ref. [8].

Table 1. Asymmetric rotational model deformation parameters are derived from $\operatorname{Ref}[8] \cdot \gamma, \gamma^{\prime}, \delta$ is in degree.

\begin{tabular}{lll}
\hline & \multicolumn{3}{c}{${ }^{56} \mathrm{Fe}$} & \\
\hline$\beta_{2}=0.2461$ & $\gamma=18.6509$ & \\
$\beta_{4}=0.046$ & $\gamma^{\prime}=3.4722$ & $\delta=173.692$ \\
\hline
\end{tabular}

For other low-lying states, up to $4.51 \mathrm{MeV}$, the vibration model is introduced. In the vibrational model, the radius is given by:

$$
R=R_{0}\left[1+\sum_{\lambda, \mu} \alpha_{\lambda}^{\mu} Y_{\lambda}^{\mu}(\theta, \phi)\right]
$$

where:

$$
\alpha_{\lambda}^{\mu}=\frac{\beta_{\lambda}^{\mu}}{\sqrt{2 \lambda+1}}\left(b_{\lambda, \mu}+(-)^{\mu} b_{\lambda,-\mu}^{+}\right) .
$$

The deformation parameters in the above formula are given in Table 2, which are adjusted to match better the experimental data.
Table 2. Vibrational model deformation parameters from Ref.[9]. Adjusted deformation parameters according to experimental data is given in last column.

\begin{tabular}{cccc}
\hline$E_{X}(\mathrm{MeV})$ & $J^{\pi}$ & $\beta_{\lambda}(N D S)$ & $\beta_{\lambda}$ \\
\hline 2.657 & $2^{+}$ & 0.0865 & 0.0690 \\
2.960 & $2^{+}$ & 0.0636 & 0.0470 \\
3.123 & $4^{+}$ & 0.1064 & 0.0895 \\
3.370 & $2^{+}$ & 0.0716 & 0.0559 \\
3.602 & $2^{+}$ & 0.0553 & 0.0450 \\
3.832 & $2^{+}$ & 0.0563 & 0.0420 \\
4.100 & $4^{+}$ & 0.0966 & 0.0966 \\
4.510 & $3^{-}$ & 0.2010 & 0.1759 \\
\hline
\end{tabular}

\section{The couple-channel Calculations}

Using the OMP parameters of An and Cai [5] as the starting point, an optimal set of couple-channel optical model potential parameters for each incident energies were determined by fitting the relevant data to minimize the quantity

$$
\chi^{2}=\frac{1}{N} \sum_{i=1}^{N}\left(\frac{\sigma_{i}^{\mathrm{cal}}-\sigma^{\mathrm{exp}}}{\Delta \sigma_{i}^{\exp }}\right)^{2}
$$

where $N$ denotes the numbers of experimental data included in the parameter search. $\Delta \sigma_{i}^{\exp }$ is the sum of the statistical and systematic errors for the data point $i$.

All experimental data are taken from the EXFOR [11] database. The distribution of experimental points is given in the Table 3, and " $\times$ " indicates the existence of experimental data. Because $11.8 \mathrm{MeV}$ is very close to $11.5 \mathrm{MeV}$, it is treated as the same incident energy in this paper. All the results are given in the center-of-mass (c.m.) system by following the experimental data set. For the experimental data without specific error, an error of $10 \%$ is set. The calculations were performed with the ECIS06 code.

Table 3. Experimental data for the parameter search.

\begin{tabular}{cccccc}
\hline$E_{X}$ & \multicolumn{5}{c}{$E_{d}(\mathrm{MeV})$} \\
$(\mathrm{MeV})$ & 11.5 & 11.8 & 12.3 & 25.9 & 56 \\
\hline 0 & & $\times$ & $\times$ & & $\times$ \\
0.846 & $\times$ & $\times$ & $\times$ & $\times$ & $\times$ \\
2.085 & $\times$ & & & & $\times$ \\
2.657 & $\times$ & & & & $\times$ \\
2.960 & $\times$ & & & & $\times$ \\
3.123 & $\times$ & & & & $\times$ \\
3.370 & $\times$ & & & & $\times$ \\
3.602 & $\times$ & & & & $\times$ \\
3.832 & $\times$ & & & & $\times$ \\
4.100 & $\times$ & & & & \\
4.510 & $\times$ & & & & $\times$ \\
\hline
\end{tabular}

We retain the original parameters for spin orbit coupling term and Coulomb term, and adjust the parameters of other terms. All modified potential parameters obtained in this way together with the starting values as given by An and Cai [5] are tabulated in Table 4, together with the relevant $\chi^{2}$ values. For the spherical OMP (denoted by "An"), only elastic scattering data is included in the calcu- 
lation of $\chi^{2}$; but for CC OMP obtained by this work (denoted by "An-C fit"), both elastic and inelastic experimental scattering data are included. There is no elastic scattering experimental data at $25.9 \mathrm{MeV}$, so the corresponding $\chi^{2}$ isn't given in Table 4. In our calculations, we do not consider the coupling among ground rotational band states and other low-lying states. We adjust the deformation parameters in the vibration model in our couple-channel calculation, based on the parameters given by DWBA method in Nuclear Data Sheets [9].

\section{Results and discussion}

Using the models and parameters mentioned above, we carried out the calculations of angular distributions for deuteron-induced elastic and inelastic scattering on ${ }^{56} \mathrm{Fe}$.

Figure 1 shows deuteron inelastic scattering angular distributions for the first low-lying state of ${ }^{56} \mathrm{Fe}$ at different incident energies. Comparing with the experimental data, the calculated results are in good agreement.

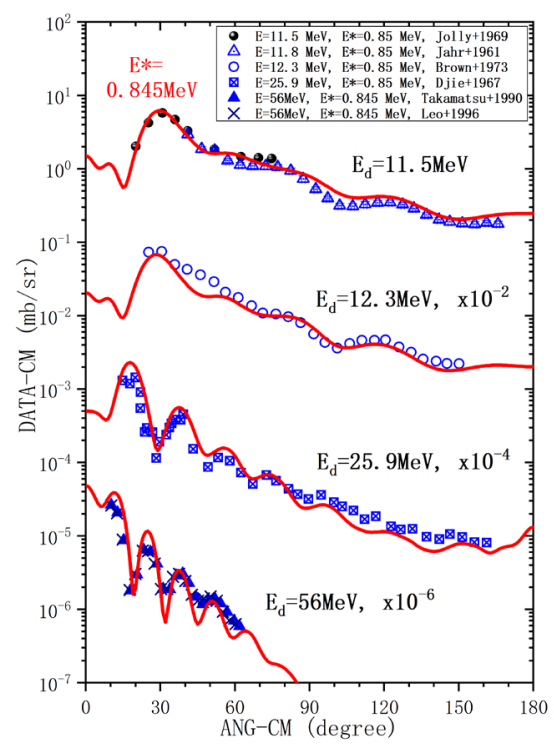

Figure 1. Comparison of deuteron inelastic scattering angular distributions with measurements for the first low-lying excited state of ${ }^{56} \mathrm{Fe}$ at different incident energies.

Figure 2 and Figure 3 show deuteron inelastic scattering angular distributions for different excited levels of ${ }^{56} \mathrm{Fe}$ at $11.5 \mathrm{MeV}$ and $56 \mathrm{MeV}$, respectively. All calculated values in Figure 2, except for $4_{1}^{+}(2.085 \mathrm{MeV})$ and $2_{3}^{+}(2.960$ $\mathrm{MeV}$ ), are in good accordance with experimental data, especially the states of spin 2. At $56 \mathrm{MeV}$ (Figure 3), the deviation between the calculated results of these two states and the experimental data is more obvious. Such big deviation has large $\chi^{2}$ at $56 \mathrm{MeV}$, and it indicates strong couplings among $4_{1}^{+}(2.085 \mathrm{MeV})$ and other states which are not included in our models yet. As for $2_{3}^{+}(2.960 \mathrm{MeV})$, Nudat 2.7 [12] shows that a state $0^{+}(2.941 \mathrm{MeV})$, is close to it. So, the experimental data may be the superposition of these two states. And it also explains why the shape of this

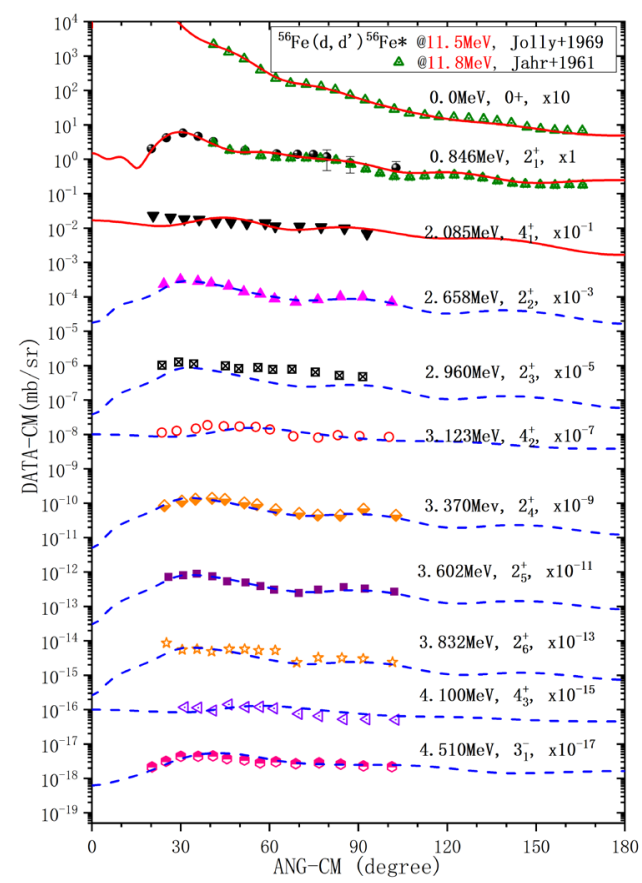

Figure 2. Comparison of deuteron inelastic scattering angular distributions with measurements for low-lying excited states of ${ }^{56} \mathrm{Fe}$ at $11.5 \mathrm{MeV}$. The solid line represents the results obtained by using the asymmetric rotational model, and the dotted line represents the results obtained by using the vibration model.

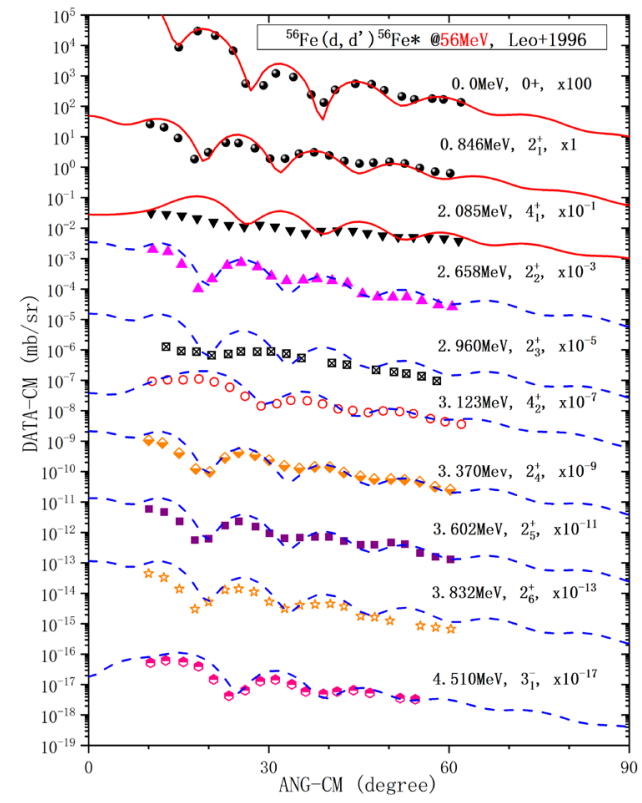

Figure 3. Comparison of deuteron inelastic scattering angular distributions with measurements for low-lying excited states of ${ }^{56} \mathrm{Fe}$ at $56 \mathrm{MeV}$. The solid line represents the results obtained by using the asymmetric rotational model, and the dotted line represents the results obtained by using the vibration model. 
Table 4. Optical model parameters derived from our best fit to the experimental data, the total reaction cross section $\sigma_{R}$ and $\chi^{2}$ of the calculation. "An" denotes the global optical potential of An and Cai. Fits with coupled channels calculations that used the potential of An and Cai as starting points are denoted by "An-C fit".

\begin{tabular}{ccccccccccccc}
\hline & $\begin{array}{c}E_{d} \\
(\mathrm{MeV})\end{array}$ & $\begin{array}{c}V_{R} \\
(\mathrm{MeV})\end{array}$ & $\begin{array}{c}r_{R} \\
(\mathrm{fm})\end{array}$ & $\begin{array}{c}a_{R} \\
(\mathrm{fm})\end{array}$ & $\begin{array}{c}W_{V} \\
(\mathrm{MeV})\end{array}$ & $\begin{array}{c}r_{V} \\
(\mathrm{fm})\end{array}$ & $\begin{array}{c}a_{V} \\
(\mathrm{fm})\end{array}$ & $\begin{array}{c}W_{S} \\
(\mathrm{MeV})\end{array}$ & $\begin{array}{c}r_{S} \\
(\mathrm{fm})\end{array}$ & $\begin{array}{c}a_{S} \\
(\mathrm{fm})\end{array}$ & $\begin{array}{c}\sigma_{R} \\
(\mathrm{mb})\end{array}$ & $\chi^{2} / N$ \\
\hline "An" & 11.5 & 93.36 & 1.150 & 0.767 & 1.82 & 1.331 & 0.472 & 10.48 & 1.374 & 0.768 & 1451 & 15.46 \\
"An-C fit" & 11.5 & 93.38 & 1.150 & 0.767 & 1.82 & 1.331 & 0.472 & 12.21 & 1.387 & 0.675 & 1388 & 3.79 \\
"An" & 12.3 & 93.17 & 1.150 & 0.767 & 1.87 & 1.331 & 0.472 & 10.45 & 1.374 & 0.768 & 1491 & 4.75 \\
"An-C fit" & 12.3 & 93.11 & 1.150 & 0.767 & 1.88 & 1.331 & 0.472 & 12.14 & 1.387 & 0.675 & 1428 & 4.66 \\
"An" & 25.9 & 89.84 & 1.150 & 0.767 & 2.71 & 1.331 & 0.472 & 10.04 & 1.374 & 0.768 & 1707 & - \\
"An-C fit" & 25.9 & 88.53 & 1.150 & 0.767 & 2.91 & 1.331 & 0.472 & 10.90 & 1.387 & 0.675 & 1641 & 12.90 \\
"An" & 56 & 82.63 & 1.150 & 0.767 & 4.59 & 1.331 & 0.472 & 9.12 & 1.374 & 0.768 & 1662 & 86.67 \\
"An-C fit" & 56 & 85.68 & 1.150 & 0.767 & 7.22 & 1.331 & 0.472 & 10.85 & 1.387 & 0.675 & 1647 & 202.86 \\
\hline
\end{tabular}

$2^{+}$state is different from other states. Further calculations are needed to confirm the above conjecture.

There are also significant deviations between the calculated results and the experimental data on $2_{4}^{+}(3.370$ $\mathrm{MeV}), 2_{5}^{+}(3.370 \mathrm{MeV})$ and $2_{6}^{+}(3.832 \mathrm{MeV})$ states, which is shown in Figure 3. We can not improve the calculation results very well by adjusting the potential parameters only. More investigation is needed.

\section{Summary}

The deuteron elastic and inelastic scattering to the first ten low-lying excited states of ${ }^{56} \mathrm{Fe}$ was analyzed in the framework of the collective motion models and by the coupled-channel method. An improved set of CC OMP parameters, and corresponding deformation parameters was deduced from the data using available global deuteron OMP parameters and deformation parameters as a starting point for the minimization of $\chi^{2}$. At low incident energies $\left(E_{d}<30 \mathrm{MeV}\right)$, except for two states, $4_{1}^{+}(2.085$ $\mathrm{MeV})$ and $2_{3}^{+}(2.960 \mathrm{MeV})$, our results are in good agreement with the experimental data. At higher incident energy $(56 \mathrm{MeV})$, it is generally consistent with the experimental data, but there are still obvious deviations for several states. The above results shows that the models used in our calculations are not enough to describe the coupling between low-lying excited states of ${ }^{56} \mathrm{Fe}$, and the further analyses and discussions are necessary.

\section{References}

[1] www.iter.org

[2] P. Garin, M. sugimoto, Fusion Eng. Des. 84, 259 (2009); V. Blideanu, et al., J. Nucl. Mater. 417, 1271 (2011); M. Garcia, et al., Fusion Sci. Technol. 62, 265 (2012).

[3] H. Xu, Y. He, P. Luo, et al., AAPPS Bull. 25(3), 3035 (2015); G. Xiao, H. Xu, S. Wang, Nucl. Phys. Rev. 34(3), 275 (2017).

[4] V. Blideanu, et al., J. Nucl. Mater. 417, 1271 (2011).

[5] H. An, C. Cai, Phys. Rev. C 73, 054605 (2006).

[6] Y. Han, et al., Phys. Rev. C 74, 044615 (2006).

[7] J. Raynal, computer program ECIS06; "Notes on ECIS94." CEA Saclay report CEA-N-2772 (1994).

[8] R.Li et al., Phys.Rev.C 87, 054611(2013).

[9] J.Huo et al., Nuclear Data Sheets 112, 1513(2011).

[10] R.De Leo et al., Phys.Rev.C 53, 2718(1996).

[11] EXchange FORmat database (EXFOR), available online at www-nds.iaea.org/exfor.

[12] NNDC (2019) NuDat Version 2.7(NuDat 2.7), available online at www.nndc.bnl.gov. 